\title{
Statistical Analysis of MIMO Beamforming With Co-Channel Unequal-Power MIMO Interferers Under Path-Loss and Rayleigh Fading
}

\author{
Yongzhao Li, Member, IEEE, Lu Zhang, Member, IEEE, Leonard J. Cimini, Jr., Fellow, IEEE, and \\ Hailin Zhang, Member, IEEE
}

\begin{abstract}
Multiple-input multiple-output (MIMO) beamforming (MBF) can greatly increase the signal gain and reduce the effects of multipath fading for cell-edge users. The objective of this work is to investigate the impact of co-channel unequal-power MIMO interferers on an MBF desired receiver under realistic propagation conditions, including path-loss and Rayleigh fading. Two major contributions are made in this work: i) for the co-channel interference term in the signal-to-interference ratio (SIR) expressions, a closed-form expression of the probability density function (PDF) is derived; ii) for the desired signal term, a simple approximation is proposed. Moreover, closed-form PDF expressions for the SIRs are obtained for some special cases. Simulation results verify the validity of the theoretical analyses.
\end{abstract}

Index Terms-Co-channel interferer, MIMO beamforming, path-loss, probability density function, signal-to-interference ratio.

\section{INTRODUCTION}

$\mathbf{C}$ O-CHANNEL interference (CCI) has a tremendous impact on cellular-like systems, especially when the goal is to use the same frequency in every cell. Multiple-input multiple-output (MIMO) based spatial diversity techniques are extensively adopted for cell-edge (or sector-edge) users to combat fading and CCI. However, when multiple-antenna techniques are incorporated, the interference scenario becomes more complicated [1]. Hence, the research related to the impact of CCI on spatial diversity has gained much attention in recent years.

Commonly used spatial diversity techniques can be classified into two categories, receive diversity and transmit diversity. Receive diversity techniques include maximal ratio combining

Manuscript received March 03, 2011; revised April 26, 2011; accepted April 27, 2011. Date of publication May 19, 2011; date of current version July 13, 2011. The associate editor coordinating the review of this manuscript and approving it for publication was Prof. Xiang-Gen Xia. The authors acknowledge the support from NSFC (61072069), RCUK for the UK-China Science Bridges Project: R\&D on (B) 4 G Wireless Mobile Communications, the Fundamental Research Funds for the Central Universities (72101855), the Important National Science \& Technology Specific Projects (2011ZX03003-001-04), the Special Grade of China Postdoctoral Science Foundation funded project (200902588), the State Key Laboratory of Integrated Services Networks (ISN090105) and the 111 project (No. B08038)

Y. Li and H. Zhang are with the State Key Laboratory of Integrated Services Networks, Xidian University, Xi'an, Shaanxi, 710071, China (e-mail: yzhli@xidian.edu.cn; hlzhang@xidian.edu.cn).

L. Zhang is with the Alcatel-Lucent Shanghai Bell Co., Ltd., Shanghai, 201206, China (e-mail: Lu.c.Zhang@ alcatel-sbell.com.cn).

L. Cimini is with the Department of Electrical and Computer Engineering, University of Delaware, Newark, DE 19716 USA (e-mail: cimini@ece.udel. edu).

Digital Object Identifier 10.1109/TSP.2011.2155654
(MRC) and optimal combining (OC) [2], which are implemented for single-input multiple-output (SIMO) systems. Transmit diversity consists of space-time block coding (STBC) [3], [4], maximal ratio transmission (MRT) and MIMO beamforming (MBF) [5]. STBC is an open-loop MIMO scheme; MBF or MRT, on the other hand, utilizes the channel state information (CSI) available at the transmitter to achieve the optimum transmission. It should be noted that MRT can be only applied in multiple-input single output (MISO) environments; so, it is often considered as a special case of MBF.

Analyzing the statistical distribution of the postprocessing signal-to-interference ratio (SIR) or signal-to-interference-plus-noise ratio (SINR) is very useful in investigating the performance of cellular-like MIMO systems. For SIMO, the SIR/SINR distribution of MRC with CCI is discussed in [6]-[11]; while the performance of OC with CCI is investigated in [9]-[11]. For a noise-limited system, MRC is well known to be the optimal combining technique in the sense of maximizing the postprocessing signal-to-noise ratio. In an interference-limited environment, OC performs optimally in terms of maximizing the SINR, but is much more complicated than MRC because it requires information about the CCI that might not be available at the receiver [10]. Thus, in practical systems, MRC is usually adopted instead of OC even in an interference-limited environment.

The performance analysis of transmit diversity techniques is much more complicated than receive diversity systems. The distribution of the postprocessing SINR for STBC with CCI was first derived in [12] and compared with other MIMO schemes, such as MRC, cyclic delay diversity, and MRT. However, in [12], it is assumed that all the base stations (BS) adopt the same MIMO mode; further, the statistical distribution is derived when equal transmit power is assumed for all interfering BSs and when the wireless channels are modeled as i.i.d. complex Gaussian random variables (RVs) with zero mean and unit variance (i.e., pure Rayleigh fading). The impact of interfering single-antenna and STBC transmissions on a desired STBC receiver is studied in [13]; however, only a simulation-based approach is used. In [14], with the channels modeled as pure Rayleigh fading, the closed-form probability density functions (PDF) of the postprocessing SIR was derived for STBC transmission when all the interfering BSs use either the same or a different MIMO mode.

For the cases where the desired BS employs MBF or MRT, which are the focus in this paper, some work has been done in 
determining or estimating the statistical distribution of the postprocessing SIR/SINR. For MBF transmission with CCI, all the existing work (e.g., see representative work in [15]-[21]) use the terminology MIMO-MRC to represent MBF and assume that each co-channel interfering signal is from a single transmit antenna. In [15], closed-form expressions for the PDF and cumulative distribution function (CDF) of the postprocessing SINR are obtained when a (2-Tx, $M$-Rx) or ( $N$-Tx, 2-Rx) antenna configuration is adopted in the desired link, and assuming all the single-antenna interferers use equal transmit power. The work in [16]-[18] derived the CDF (equivalently, outage probability) and/or PDF of the postprocessing SIR/SINR when an $(N-\mathrm{Tx}$, $M$-Rx) antenna configuration is adopted in the desired link for unequal-power interferers. Using the same model for the desired and interfering links as in [16]-[18], the work in [19]-[21] analyzed the distribution of the postprocessing SINR with imperfect channel estimation.

When MBF is in used in the desired link, for the cases where each interfering BS performs single-antenna transmission and each interfering BS adopts MBF/MRT to perform multi-antenna transmission, the same statistical distribution of the postprocessing SIR results. However, it will be shown in this paper that, if each interfering BS adopts STBC, open-loop spatial multiplexing (OLSM), or closed-loop spatial multiplexing (CLSM), the resulting statistical distribution of the postprocessing SIR will be distinct from the case in which each interfering BS adopts MBF/MRT/SIMO; but, for these cases, no work has been done previously.

In this paper, in order to provide a comprehensive investigation of the impact of each possible co-channel MIMO mode on an MBF receiver, taking into account the effects of both path-loss and Rayleigh fading, we derive the distributions of the postprocessing SIRs (noise is neglected here since we are considering an interference-limited environment) for MBF with different co-channel MIMO modes and possibly unequal-power interferers. In particular, closed-form PDF expressions for the interference terms in the postprocessing SIRs are provided. The validity of the theoretical analyses is verified by simulation results.

The system model and notations are described in Section II. The postprocessing SIR expressions are derived in Section III. Then, we present the PDF of the SIR in Section IV. Simulation results and conclusions are provided in Sections V and VI, respectively.

\section{System Model AND NotATIONS}

A typical interference-limited system using MBF in the desired link with $K$ co-channel interfering BSs is considered in this paper. Without loss of generality, six widely applied MIMO modes: STBC, OLSM, CLSM, MRT, MBF, and SIMO are considered for the co-channel interfering BSs. Each transmitter and the desired receiver are assumed to be equipped with $N$ and $M$ antennas, respectively. The channel between the $k$ th $(k=$ $0,1, \ldots, K) \mathrm{BS}$ and the desired receiver is denoted by

$$
\mathbf{H}_{k}=\left[h_{k, j i}\right]_{M \times N}
$$

where $h_{k, j i}(j=1, \ldots, M ; i=1, \ldots, N)$ represents the instantaneous channel coefficient between the $j$ th receiving antenna at the desired user and the $i$ th transmitting antenna at the $k$ th BS. The index $k=0$ indicates the desired BS. Note that, when SIMO (i.e., $N=1$ ) is used for the $k$ th interfering BS, the channel matrix between the interfering BS and the desired receiver becomes an $M \times 1$ vector. Here, we use the first column of $\mathbf{H}_{k}$ to denote it.

The channel coefficients $\left\{h_{k, j i}\right\}$ include the effects of pathloss and quasi-static flat Rayleigh fading. Under these assumptions, since the distances from the antennas of a co-located array to the antennas of another co-located array can be considered equal, for any given $k$, the coefficients $\left\{h_{k, j i}\right\}$ have the same mean channel gain $\mu_{k}$. It is assumed that the system is located in a rich scattering environment and the antennas are sufficiently separated; so, the channels $\left\{h_{k, j i}\right\}$ can be modeled as independent, complex Gaussian RVs with zero mean and variance $\mu_{k}$. It should be noted that both the effects of path-loss and Rayleigh fading are included in $\mu_{k}$. Moreover, the transmit power of each antenna at the $k$ th $\mathrm{BS}$ is denoted as $P_{k}$. For simplicity, we include the transmit powers in the channel power gains; so, we consider an equivalent system in which i) the channels $\left\{h_{k, j i}\right\}$ are independent, complex Gaussian RVs with zero mean and variance $P_{k} \mu_{k}$ and ii) each antenna at each BS uses unit power. Here, $P_{k} \mu_{k}$ is the average received power at one receive antenna (of the desired user) from one transmit antenna (of the $k$ th BS). Since path-loss and transmit power may be distinct for different BSs, $P_{i} \mu_{i}$ may not be equal to $P_{j} \mu_{j}(i \neq j)$.

Since we consider an interference-limited environment, the effect of noise will be neglected in the subsequent analysis. We assume that full CSI of the desired link (i.e., $\mathbf{H}_{0}$ ) is available at the desired BS. We also assume that the network is de-centralized, i.e., no knowledge of the interferers can be utilized by the desired receiver and the transmissions of the different BSs are independent. This can be considered as a "worst-case" scenario.

Notations: The superscripts $*, T$, and $H$ denote complex conjugate, transpose and conjugate transpose, respectively. $E[\cdot]$ denotes statistical expectation; $\mathbf{I}_{N}$ is the $N \times N$ identity matrix; $\|\mathbf{a}\|$ denotes the norm of a complex valued vector $\mathbf{a}$, i.e., $\|\mathbf{a}\|=\sqrt{\mathbf{a}^{H} \mathbf{a}} ; \mathbb{C}^{m \times n}$ denotes the vector space of all $m \times n$ complex matrices; $\mathbf{A}(j,:)$ and $\mathbf{A}(:, i)$ denote the $j$ th row and the $i$ th column of matrix $\mathbf{A}$, respectively; $\mathcal{C N}\left(\mu, \sigma^{2}\right)$ denotes the circularly symmetric complex Gaussian distribution with mean $\mu$ and variance $\sigma^{2}$; and $\Gamma(\cdot)$ denotes the gamma function.

\section{POSTPROCESSING SIR}

In this section, for the case where the desired link adopts $\mathrm{MBF}$, we derive and analyze expressions for the postprocessing SIR considering the special case where all the interfering BSs adopt the same MIMO mode. Based on the analyses in this section, the six typical MIMO modes can be effectively classified into two categories of interfering MIMO modes. Then, with the help of this classification, the subsequent derivation for the SIR distribution can be greatly simplified.

In the desired link, which adopts MBF (assuming $\mathbf{H}_{0}$, as defined in (1), is perfectly known at the desired BS), $s_{0}$ is precoded 
by a vector $\mathbf{w}_{0} \in \mathbb{C}^{N \times 1}$ before transmission. At the desired receiver, the baseband received vector $\mathbf{r} \in \mathbb{C}^{M \times 1}$, can be modeled as

$$
\mathbf{r}=\mathbf{H}_{0} \mathbf{w}_{0} s_{0}+\sum_{k=1}^{K} \mathbf{H}_{k} \mathbf{w}_{k} \mathbf{s}_{k}
$$

where $\mathbf{w}_{k}$ and $\mathbf{s}_{k}$ are the precoding matrix (or vector) and the transmitted symbol(s), respectively, for the $k$ th interfering BS. The sizes and natures of $\mathbf{w}_{k}$ and $\mathbf{s}_{k}$ depend on the specific MIMO mode used in the $k$ th interfering BS, which will be explained in what follows.

\section{A. Desired User Has One Receive Antenna}

When the desired user has only one receive antenna (i.e., $M=1$ ), as a special case of MBF, MRT [5] is adopted at the desired BS. In this scenario, $\mathbf{H}_{0}$ reduces to a $1 \times N$ vector and the precoding vector for the desired link is defined as

$$
\mathbf{w}_{0}=\frac{\sqrt{N} \mathbf{H}_{0}^{H}}{\left\|\mathbf{H}_{0}\right\|}
$$

For simplicity, we assume $E\left[\left|s_{0}\right|^{2}\right]=1$, although this assumption can be easily relaxed. Using (2) and (3), the average received power of the desired signal $s_{0}$ can be computed as

$$
\left|\mathbf{H}_{0} \mathbf{w}_{0}\right|^{2}=N \cdot\left\|\mathbf{H}_{0}\right\|^{2}=N \cdot \sum_{i=1}^{N}\left|h_{0,1 i}\right|^{2} .
$$

Next, we analyze the average interfering power and obtain the SIR expressions for different MIMO modes used in the interfering links.

1) Interfering Links Use STBC or OLSM: When all the interfering BSs adopt STBC or OLSM, no precoding is used in the interfering links, i.e., $\mathbf{w}_{k}=\mathbf{I}_{N} \cdot \mathbf{s}_{k}$, which consists of $N$ independent symbols of the form

$$
\mathbf{s}_{k}=\left[s_{k, 1}, \ldots, s_{k, N}\right]^{T}
$$

is sent simultaneously from the $k$ th interfering BS. By assuming $E\left[\left|s_{k, i}\right|^{2}\right]=1(i=1, \ldots, N)$ and exploiting the independence of $\left\{h_{k, 1 i}\right\}$, the average interference power corrupting $s_{0}$ can be computed as

$$
\sum_{k=1}^{K}\left\|\mathbf{H}_{k} \mathbf{w}_{k}\right\|^{2}=\sum_{k=1}^{K}\left\|\mathbf{H}_{k}\right\|^{2}=\sum_{i=1}^{N} \sum_{k=1}^{K}\left|h_{k, 1 i}\right|^{2}
$$

where $\mathbf{H}_{k} \in \mathbb{C}^{1 \times N}$ is defined by (1) with $M=1$ and $h_{k, 1 i} \sim$ $\mathcal{C N}\left(0, P_{k} \mu_{k}\right)$.

2) Interfering Links Use CLSM: When all the interfering BSs adopt CLSM, $\mathbf{s}_{k}$, with the same definition as (5), is precoded before being sent simultaneously from the $k$ th interfering BS. Assuming that the CSI of the $k$ th intra-cell link is available at the $k$ th interfering BS, the precoding unitary matrix $\mathbf{w}_{k} \in \mathbb{C}^{N \times N}$ is obtained through a singular value decomposition (SVD) of the $k$ th intra-cell channel matrix. By integrating the precoding block into the $k$ th CCI channel, we can establish an equivalent model for the channel vector between the $k$ th interfering BS and the desired receiver which has the form

$$
\widetilde{\mathbf{h}}_{k} \triangleq \mathbf{H}_{k} \mathbf{w}_{k}=\left[\mathbf{H}_{k} \mathbf{w}_{k}(:, 1), \ldots, \mathbf{H}_{k} \mathbf{w}_{k}(:, N)\right]
$$

Therefore, the average interfering power can be evaluated as

$$
\sum_{k=1}^{K}\left\|\mathbf{H}_{k} \mathbf{w}_{k}\right\|^{2}=\sum_{k=1}^{K}\left\|\widetilde{\mathbf{h}}_{k}\right\|^{2}=\sum_{i=1}^{N} \sum_{k=1}^{K}\left|\mathbf{H}_{k} \mathbf{w}_{k}(: ; i)\right|^{2} .
$$

Because $\mathbf{w}_{k}$ is an SVD-generated unitary matrix and independent of $\mathbf{H}_{k},\left\{\mathbf{H}_{k} \mathbf{w}_{k}(:, i)\right\}$ are still independent RVs with distribution $\mathcal{C N}\left(0, P_{k} \mu_{k}\right)$. By comparing $\mathbf{H}_{k} \mathbf{w}_{k}(:, i)$ with $h_{k, 1 i}$ in (6), which also follows $\mathcal{C N}\left(0, P_{k} \mu_{k}\right)$, we find that (8) and (6) have the same distribution.

3) Interfering Links Use MBF or MRT: When MBF or MRT is used by the interfering BSs, $\mathbf{s}_{k}$ reduces to a scalar, $s_{k}$. In other words, the $N$ transmit antennas are used to send the same symbol during one symbol period. In the case of MBF, the precoding unitary vector $\mathbf{w}_{k} \in \mathbb{C}^{N \times 1}$ is constructed from the unitary singular vector, $\mathbf{v}_{k, 1}$, which corresponds to the maximum singular value of the $k$ th intra-cell channel matrix, $\mathbf{G}_{k}$. To satisfy the same transmit power constraint as for other MIMO modes, we define

$$
\mathbf{w}_{k}=\sqrt{N} \mathbf{v}_{k, 1} \text { with }\left\|\mathbf{v}_{k, 1}\right\|^{2}=1 \text {. }
$$

In the case of MRT, the precoding unitary vector is

$$
\mathbf{w}_{k}=\frac{\sqrt{N} \mathbf{g}_{k}^{H}}{\left\|\mathbf{g}_{k}\right\|} \triangleq \sqrt{N} \mathbf{v}_{k, 1}
$$

where $\mathrm{g}_{k} \triangleq\left[g_{k, 1}, \ldots, g_{k, N}\right]^{T}$ is the $k$ th intra-cell channel vector. Note that, the intra-cell link precoding vectors for MBF and MRT have the same norm $\sqrt{N}$, which means (9) and (10) have the same functionality when they act as the precoding vectors. Hence, MBF and MRT have the same performance when they are used in the interfering BSs. Using the notation in (9) and the assumption of $E\left[\left|s_{k}\right|^{2}\right]=1$, the average power of the interference on $s_{0}$ can be written as

$$
\sum_{k=1}^{K}\left|\mathbf{H}_{k} \mathbf{w}_{k}\right|^{2}=N \cdot \sum_{k=1}^{K}\left|\mathbf{H}_{k} \mathbf{v}_{k, 1}\right|^{2}=N \cdot \sum_{k=1}^{K}\left|\widetilde{h}_{k, 11}\right|^{2}
$$

where $\widetilde{h}_{k, 11} \triangleq \mathbf{H}_{k} \mathbf{w}_{k}$ represents the equivalent channel between the $k$ th interfering BS and the desired receiver. Since $\mathbf{v}_{k, 1}$ (inter-cell link precoding vector) is unitary and independent of $\mathbf{H}_{k}$ (inter-cell link channel vector), $\widetilde{h}_{k, 11}$ is also distributed as $\mathcal{C N}\left(0, P_{k} \mu_{k}\right)$.

4) Interfering Links Use SIMO: Note that, when SIMO and MRT are used for the interfering BSs and desired BS, $\mathbf{H}_{k}$ and $\mathbf{s}_{k}$ reduce to the scalars $h_{k, 11}$ and $s_{k}$, respectively. Moreover, to satisfy the same transmit power constraint as for the other MIMO modes, we define

$$
\mathbf{w}_{k}=\sqrt{N}
$$


as a normalized power factor. Similar to other MIMO modes, we assume $E\left[\left|s_{k}\right|^{2}\right]=1$. Hence, the average power of the interference on $s_{0}$ is

$$
\sum_{k=1}^{K}\left|\mathbf{H}_{k} \mathbf{w}_{k}\right|^{2}=N \cdot \sum_{k=1}^{K}\left|h_{k, 11}\right|^{2} .
$$

Since $h_{k, 11}$ follows the distribution $\mathcal{C N}\left(0, P_{k} \mu_{k}\right),(13)$ and (11) have the same distribution.

Based on the above analysis, we divide the six co-channel MIMO modes into the following two categories when MRT is used in the desired link:

- Category I: STBC, OLSM, and CLSM;

- Category II: MBF, MRT, and SIMO.

We conclude that the SIRs of the same category have the same statistical distribution. In other words, the same category has the same effect on the desired received signal. Therefore, we can obtain the SIR of the received signal at the desired receiver as

$$
\gamma_{\mathrm{MRT}, \mathrm{I}}=\frac{\sum_{i=1}^{N}\left|h_{0,1 i}\right|^{2}}{\frac{1}{N} \cdot \sum_{i=1}^{N} \sum_{k=1}^{K}\left|h_{k, 1 i}\right|^{2}}
$$

for Category I; and

$$
\gamma_{\mathrm{MRT}, \mathrm{II}}=\frac{\sum_{i=1}^{N}\left|h_{0,1 i}\right|^{2}}{\sum_{k=1}^{K}\left|h_{k, 11}\right|^{2}}
$$

for Category II. For simplicity, we rewrite (14) and (15) as

$$
\gamma_{\mathrm{MRT}}=\frac{\sum_{i=1}^{N}\left|h_{0,1 i}\right|^{2}}{\frac{1}{N^{2-q}} \cdot \sum_{i=1}^{N^{2-q}} \sum_{k=1}^{K}\left|h_{k, 1 i}\right|^{2}}
$$

where $q$ ( $q=1$ for Category I or $q=2$ for Category II) is an index representing the category of the MIMO mode of the interference; $h_{0,1 i}$ and $h_{k, 1 i}$ are independent and follow $\mathcal{C N}\left(0, P_{0} \mu_{0}\right)$ and $\mathcal{C N}\left(0, P_{k} \mu_{k}\right)$, respectively.

Since $h_{0,1 i} \sim \mathcal{C N}\left(0, P_{0} \mu_{0}\right)$, it is easy to show that the numerator $X \triangleq \sum_{i=1}^{N}\left|h_{0,1 i}\right|^{2}$ in (16) follows a central chi-square distribution with PDF

$$
f_{X}(x)=\frac{x^{N-1} \exp \left\{\frac{-x}{\left(P_{0} \mu_{0}\right)}\right\}}{\left(P_{0} \mu_{0}\right)^{N} \Gamma(N)} .
$$

Similarly, we can show that $y_{k, i} \triangleq\left|h_{k, 1 i}\right|^{2}(k=1, \ldots, K ; i=$ $1, \ldots, N)$ is an exponential distributed $\mathrm{RV}$ with $\mathrm{PDF}$

$$
f_{y_{k, i}}\left(y_{k, i}\right)=\frac{1}{P_{k} \mu_{k}} \exp \left\{\frac{-y_{k, i}}{\left(P_{k} \mu_{k}\right)}\right\} .
$$

Moreover, the numerator and the denominator in (16) are obviously independent. So, once the distribution of the denominator is determined, the PDF of the SIR can be obtained by using the independence feature.

\section{B. SIR Expression for General $N$ and $M$}

Here, we consider general $N$ and $M(\min \{N, M\} \geq 2)$. Assume that the channel matrix $\mathbf{H}_{0} \in \mathbb{C}^{M \times N}$ is perfectly known at the desired BS. To obtain the precoding unitary vector for MBF, the desired BS implements an SVD on $\mathbf{H}_{0}$; thus,

$$
\mathbf{H}_{0}=\mathbf{U}_{0} \mathbf{D}_{0} \mathbf{V}_{0}^{H}
$$

where $\mathbf{U}_{0} \in \mathbb{C}^{M \times M}$ and $\mathbf{V}_{0} \in \mathbb{C}^{N \times N}$ are the left and right unitary matrices, respectively; $\mathbf{D}_{0} \in \mathbb{C}^{M \times N}$ is a rectangular diagonal matrix with $\min \{N, M\}$ non-negative real singular values $d_{0,1}, \ldots, d_{0, \min \{N, M\}}$. Without loss of generality, we assume $d_{0,1} \geq d_{0,2} \geq \cdots \geq d_{0, \min \{N, M\}-1} \geq d_{0, \min \{N, M\}}$. Specifically, for the largest singular value $d_{0,1}$, the corresponding left and right singular vectors are denoted by $\mathbf{u}_{0,1} \in \mathbb{C}^{M \times 1}$ and $\mathbf{v}_{0,1}^{H} \in \mathbb{C}^{1 \times N}$, respectively.

At the desired transmitter, $s_{0}$ is precoded by the beamforming vector $\mathbf{w}_{0}=\sqrt{N} \mathbf{v}_{0,1}$, and then transmitted. At the desired receiver, the received signal is multiplied by $\mathbf{u}_{0,1}^{H}$ to recover $s_{0}$. Hence, the postprocessing received signal at the desired user is

$$
\begin{aligned}
\mathbf{u}_{0,1}^{H} \mathbf{r} & =\mathbf{u}_{0,1}^{H} \mathbf{H}_{0} \mathbf{w}_{0} s_{0}+\sum_{k=1}^{K} \mathbf{u}_{0,1}^{H} \mathbf{H}_{k} \mathbf{w}_{k} \mathbf{s}_{k} \\
& =\sqrt{N} d_{0,1} s_{0}+\sum_{k=1}^{K} \mathbf{u}_{0,1}^{H} \mathbf{H}_{k} \mathbf{w}_{k} \mathbf{s}_{k} .
\end{aligned}
$$

Then, we obtain the average desired signal power in the postprocessing received signal as

$$
N \cdot\left|d_{0,1}\right|^{2} \text {. }
$$

Similar to Section III-A (MRT in the desired link), by computing and comparing the average interfering power for different interfering MIMO modes, the six co-channel MIMO modes can also be classified into two categories when MBF is used in the desired link. As a result, the following postprocessing SIR expression can be obtained:

$$
\gamma_{\mathrm{MBF}}=\frac{\left|d_{0,1}\right|^{2}}{\frac{1}{N^{2-q}} \cdot \sum_{i=1}^{N^{2-q}} \sum_{k=1}^{K}\left|\widetilde{h}_{k, 1 i}\right|^{2}}
$$

where $\widetilde{h}_{k, 1 i}$ (depends on $\mathbf{u}_{0,1}, \mathbf{H}_{k}$ and $\mathbf{w}_{k}$ ) is i.i.d. complex Gaussian distributed and follows $\mathcal{C N}\left(0, P_{k} \mu_{k}\right)$. Obviously, $\widetilde{y}_{k, i} \triangleq\left|\widetilde{h}_{k, 1 i}\right|^{2}(k=1, \ldots, K ; i=1, \ldots, N)$ follows an exponential distribution with the same PDF in (18).

Since $\mathbf{H}_{0}=\mathbf{U}_{0} \mathbf{D}_{0} \mathbf{V}_{0}^{H}$ is a random matrix with i.i.d. complex Gaussian entries, it is isotropically random (i.e., spherically symmetric). This implies $\mathbf{U}_{0}, \mathbf{D}_{0}$ and $\mathbf{V}_{0}$ are independent of each other [22]. Note that, the numerator and the denominator in (22) correspond to $d_{0,1}\left(\mathbf{D}_{0}\right)$ and $\mathbf{u}_{0,1}\left(\mathbf{U}_{0}\right)$, respectively. Additionally, using the independence of $\mathbf{H}_{0}$ and $\mathbf{H}_{k}$, we conclude that the numerator and the denominator are mutually independent.

Next, we derive the PDFs of $\left|d_{0,1}\right|^{2}$ (for $\min \{N, M\} \geq 2$ ), the denominators, and the corresponding SIRs when MBF is adopted for the desired link. Furthermore, it should be noted that the denominators in (16) and (22) have the same statistical distribution. Hence, the PDF of the denominator in (22) can also be used to determine the SIR distribution for MRT in the desired link. 


\section{SIR DISTRIBUTION}

\section{A. Distribution of $\left|d_{0,1}\right|^{2}$}

The distribution of $\left|d_{0,1}\right|^{2}$ can be obtained by evaluating the distribution of the largest eigenvalue of the Wishart matrix $\mathbf{H}_{0}^{H} \mathbf{H}_{0} \in \mathbb{C}^{N \times N}$. (Note that $\mathbf{H}_{0}^{H} \mathbf{H}_{0}$ and $\mathbf{H}_{0} \mathbf{H}_{0}^{H}$ have identical eigenvalues; so, without loss of generality, we use $\mathbf{H}_{0}^{H} \mathbf{H}_{0}$ as the example here.) Using (19), we get the following eigenvalue decomposition of $\mathbf{H}_{0}^{H} \mathbf{H}_{0}$ :

$\mathbf{H}_{0}^{H} \mathbf{H}_{0}=\mathbf{V}_{0} \operatorname{diag}\left(\left|d_{0,1}\right|^{2}, \ldots,\left|d_{0, \min \{N, M\}}\right|^{2}, 0, \ldots, 0\right) \mathbf{V}_{0}^{H}$.

Clearly, the largest eigenvalue $\lambda_{\max }$ is $\left|d_{0,1}\right|^{2}$. Let $t=$ $\min \{N, M\}$ and $r=\max \{N, M\}$; the PDF of $\lambda_{\max }$ is [23]

$$
\begin{aligned}
f_{\lambda_{\max }}(\lambda)=\frac{1}{\prod_{i=1}^{t}(t-i) !(r-i) !} \\
\quad \times \sum_{i=1}^{t} \sum_{m=r-t}^{(t+r) i+2 i^{2}} c_{i, m} e^{-i \lambda} \lambda^{m}, \lambda>0
\end{aligned}
$$

where $c_{i, m}$, the coefficient of $e^{-i \lambda} \lambda^{m}$, can be evaluated using symbolic computation software [23]. Due to the unavailability of closed-form expressions for $c_{i, m}$, a usable expression for (24) cannot be obtained. Therefore, we need to make some reasonable approximations to the distribution of $\left|d_{0,1}\right|^{2}$ (i.e., $\lambda_{\max }$ ).

Using the same classification of the interfering MIMO modes, the postprocessing SIR when STBC is used for the desired link with $K q^{\text {th }}(q=1,2)$ category interferers can be written as [14]

$$
\gamma_{\mathrm{STBC}} \approx \frac{1}{N} \cdot \frac{x_{0}}{\frac{1}{N^{2-q}} \sum_{i=1}^{N^{2-q}} \sum_{k=1}^{K} y_{k, i}}
$$

where $x_{0}$ follows a chi-square distribution with degree $2 N M$, and $\left\{y_{k, i}\right\}\left(k=1, \ldots, K ; i=1, \ldots, N^{2-q}\right)$ are independent exponential RVs with PDF $f_{y_{k, i}}\left(y_{k, i}\right)=\frac{1}{P_{k} \mu_{k}} \exp \left\{\frac{-y_{k, i}}{\left(P_{k} \mu_{k}\right)}\right\}$. By comparing (22) with (25), we can see that the distributions of the denominators (co-channel interference terms) in the SIRs for $\mathrm{MBF}$ and STBC are the same. The numerators (desired signal terms) are different, however. In the case of STBC, the numerator $x_{0}$ follows a chi-square distribution with degree $2 N M$, which corresponds to the diversity gain $N M$ that the desired received signal can obtain. For an ( $N$-Tx, $M$-Rx) MIMO channel, MBF can also achieve the maximum spatial diversity gain of order $N M$ [24]-[26]. Hence, for an ( $N-$ Tx, $M$-Rx) antenna configuration, both STBC and MBF are equivalent to a receive diversity system with $N M$ receive antennas. This is an important similarity. Since a receive diversity system with $N M$ receive antennas using MRC achieves diversity order $N M$, the distribution for the desired signal term of MRC (the sum of $N M$ square moduli of independent channel coefficients) is chisquare. Hence, it is reasonable to approximate the distribution of $\lambda_{\text {max }}$ (desired signal term for MBF) as a chi-square RV with degree $2 N M$. The validity of this approximation will be justified by Monte Carlo simulations in Section V.

Besides this similarity, we also need to consider the differences between these two MIMO schemes. Although STBC and MBF can achieve the same diversity gain, MBF cannot utilize the full channel energy to transmit the signal. By implementing
SVD (transmit precoding) at the transmitter and shaping at the receiver, an ( $N$-Tx, $M$-Rx) MIMO channel can be converted into $\min \{N, M\}$ independent channels. Different from other closed-loop MIMO schemes (e.g., CLSM), MBF can only utilize the channel with the largest gain (corresponding to $\lambda_{\max }$ ) to transmit the signals. Hence, the channel power provided by MIMO cannot be fully utilized by MBF. Rather, part of the channel power is inevitably consumed by channels with gains corresponding to the smaller eigenvalues.

Therefore, a reasonable approximate statistical model for $\lambda_{\max }$ must take the loss of channel power into account. Because the power of every decoupled channel is proportional to its corresponding eigenvalue in the Wishart matrix, we define the percentage of energy achieved by the channel with gain $\sqrt{\lambda_{\max }}$ as

$$
\zeta=\frac{\mathrm{E}\left[\lambda_{\max }\right]}{\mathrm{E}\left[\sum_{i=1}^{\min \{N, M\}} \lambda_{i}\right]}
$$

where $\left\{\lambda_{i}\right\}(i=1, \ldots, \min \{N, M\})$ are the nonzero eigenvalues of the Wishart matrix $\mathbf{H}_{0}^{H} \mathbf{H}_{0}$. Using (1), (19), and (23), the denominator in (26) is

$$
\begin{aligned}
E\left[\sum_{i=1}^{\min \{N, M\}} \lambda_{i}\right] & =E\left[\operatorname{tr}\left\{\mathbf{H}_{0}^{H} \mathbf{H}_{0}\right\}\right] \\
& =E\left[\sum_{j=1}^{M} \sum_{i=1}^{N}\left|h_{0, j i}\right|^{2}\right] \\
& =N M \cdot P_{0} \mu_{0}
\end{aligned}
$$

where $\operatorname{tr}\{\cdot\}$ denotes the trace of a matrix. Here, we use the fact that $\left\{h_{0, j i}\right\}(j=1, \ldots, M ; i=1, \ldots, N)$ are i.i.d. complex Gaussian RVs and $\mathrm{E}\left[\left|h_{0, j i}\right|^{2}\right]=P_{0} \mu_{0}$.

The numerator in (26) requires the expectation of $\lambda_{\max }$. Although (24) can be used, it is difficult. An alternative approach is to use some empirical formulas; specifically, as shown in [27], we can use the following expression to evaluate the mean of the largest eigenvalue of the Wishart matrix for an $(N-\mathrm{Tx}, M-\mathrm{Rx})$ antenna configuration

$$
E\left[\lambda_{\max }\right] \approx N M\left(\frac{N+M}{N M+1}\right)^{\frac{2}{3}} \cdot P_{0} \mu_{0}, N M \leq 250 .
$$

When $M=1$ (MRT in the desired link), from (26), (27), and (28), $\zeta=1$. This means that all of the channel energy is concentrated in one channel, and no energy is lost. Also note that (28) is symmetric in $N$ and $M$, and the mean of $\lambda_{\max }$ depends on $N+M$ and $N M$.

Based on the above analysis, we propose an approximate distribution for the largest eigenvalue $\lambda_{\max }$. Using the fact that MBF can achieve the maximum diversity gain $N M$, and, with the aid of the introduction of $\zeta$, we model the largest eigenvalue of the Wishart matrix $\mathbf{H}_{0}^{H} \mathbf{H}_{0}$ as a chi-square distributed $\mathrm{RV}$ (with degree $2 N M$ ) multiplied by the coefficient $\zeta$. The resulting PDF is

$$
f_{\lambda_{\max }}(\lambda) \approx \frac{\lambda^{N M-1}}{\Gamma(N M)\left(\zeta P_{0} \mu_{0}\right)^{N M}} e^{-\frac{\lambda}{\zeta P_{0} \mu_{0}}}, \lambda>0 .
$$


Note that, although (29) is derived for $\min \{N, M\} \geq 2$ which corresponds to MBF in the desired link, it agrees with (17) for $M=1$ (i.e., $\zeta=1$ ) which corresponds to MRT in the desired link.

\section{B. Distribution of Denominator in SIR}

By comparing (16) and (22), we see that the corresponding denominators have the same distribution. Without loss of generality, the notations in (16) are used in the following derivations. We rewrite the denominator (excluding the coefficient $N^{2-q}$ ) of (16) as

$$
Y \triangleq \sum_{i=1}^{N^{2-q}} \sum_{k=1}^{K}\left|h_{k, 1 i}\right|^{2}=\sum_{i=1}^{N^{2-q}} \sum_{k=1}^{K} y_{k, i}
$$

in which $y_{k, i} \triangleq\left|h_{k, 1 i}\right|^{2}$ follows a chi-square distribution with degree 2 (i.e., exponential distribution) and its PDF is $f_{y_{k, i}}\left(y_{k, i}\right)=\frac{1}{P_{k} \mu_{k}} \exp \left\{\frac{-y_{k, i}}{\left(P_{k} \mu_{k}\right)}\right\}$. Denote $\alpha_{l}\left(l=1, \ldots, K N^{2-q}\right)$ as independent zero-mean complex Gaussian RVs with variances $P_{k} \mu_{k}\left(k=\left\lceil\frac{l}{N^{2-q}}\right\rceil\right)$, where $\left\lceil\frac{l}{N^{2-q}}\right\rceil$ means "rounding $\frac{l}{N^{2-q}}$ to the next largest integer". Then, we can rewrite $y_{k, i}$ as

$$
y_{k, i}=\left|\alpha_{(k-1) N^{2-q}+i}\right|^{2}
$$

where $k=1, \ldots, K ; i=1, \ldots, N^{2-q}$. Let $\alpha=$ $\left[\alpha_{1}, \ldots, \alpha_{K N^{2-q}}\right]^{T}$ and denote the $K N^{2-q} \times K N^{2-q}$ identity matrix as $\mathbf{I}_{K N^{2-q}}$. Because the identity matrix $\mathbf{I}_{K N^{2-q}}$ is a semi-positive definite Hermitian matrix, and $\boldsymbol{\alpha}$ is a zero-mean complex Gaussian random vector, $\sum_{i=1}^{N^{2-q}} \sum_{k=1}^{K}\left|h_{k, 1 i}\right|^{2}=$ $\sum_{i=1}^{N^{2-q}} \sum_{k=1}^{K} y_{k, i}=\boldsymbol{\alpha}^{H} \boldsymbol{\alpha}=\boldsymbol{\alpha}^{H} \mathbf{I}_{K N^{2-q}} \boldsymbol{\alpha}$ is a central quadratic form in complex Gaussian RVs [28]. Further, denote the covariance matrix of $\boldsymbol{\alpha}$ as $\mathbf{R} \in \mathbb{C}^{K N^{2-q} \times K N^{2-q}}$ which can be written as (32), shown at the bottom of the page. Because $\mathbf{R}$ is a semi-positive definite real symmetric matrix, there exists a real symmetric matrix $\mathbf{Q} \in \mathbb{C}^{K N^{2-q} \times K N^{2-q}}$ for which $\mathbf{Q Q}=\mathbf{R}$. We can easily obtain (33) at the bottom of the page. Denote $\mathbf{B}$ as $\mathbf{Q} \mathbf{I}_{K N^{2-}-q} \mathbf{Q}$, which is a semi-positive definite Hermitian matrix. Since $\mathbf{B}=\mathbf{Q} \mathbf{I}_{K N^{2-} q} \mathbf{Q}=\mathbf{R}$, the eigenvalue decomposition of $\mathbf{B}$ is equal to the eigenvalue decomposition of $\mathbf{R}$. That is to say, the $K N^{2-q}$ eigenvalues of $\mathbf{B}$ are

$$
\lambda_{(k-1) N^{2-q}+i}=P_{k} \mu_{k}
$$

where $k=1, \ldots, K ; i=1, \ldots, N^{2-q}$. Without loss of generality, denote $M_{p}\left(M_{p} \leq K N^{2-q}\right)$ as the total number of distinct positive eigenvalues and $v_{m}$ as the multiplicity of $\lambda_{m}(m=$ $\left.1, \ldots, M_{p}\right)$. For central quadratic forms in complex Gaussian $\mathrm{RVs}$, the moment generating function (MGF) of $Y$ can be expressed as [28]

$$
\Phi_{Y}(s)=E\left[e^{s y}\right]=\frac{1}{\prod_{m=1}^{M_{p}}\left(1-s \lambda_{m}\right)^{v_{m}}} .
$$

Denote $f_{Y}(y)$ as the PDF of $Y$, which is the inverse Laplace transform of $\Phi_{Y}(s)$. Since the value of $Y$ is definitely non-negative, we derive $f_{Y}(y)$ for $y \geq 0$. By utilizing the Laplace inversion theorem, Jordan's lemma, and the residue theorem [29], we get

$$
\begin{aligned}
f_{Y}(y) & =-\sum_{m=1}^{M_{p}} f_{m}(y) \\
& =-\sum_{m=1}^{M_{p}} \operatorname{Res}\left[\Phi_{Y}(s) e^{-s y}, \frac{1}{\lambda_{m}}\right], \quad y \geq 0
\end{aligned}
$$

where $f_{m}(y)$ is the residue of $\Phi_{Y}(s) e^{-s y}$ at the pole $\frac{1}{\lambda_{m}}$. Then, we calculate $f_{m}(y)\left(m=1, \ldots, M_{p}\right)$ as follows:

$$
\begin{aligned}
f_{m}(y)= & \operatorname{Res}\left[\Phi_{Y}(s) e^{-s y}, \frac{1}{\lambda_{m}}\right] \\
= & \frac{1}{\Gamma\left(v_{m}\right)} \lim _{s \rightarrow \frac{1}{\lambda_{m}}} \frac{d^{v_{m}-1}}{d s^{v_{m}-1}} \\
& \times\left[\Phi_{Y}(s) e^{-s y}\left(s-\frac{1}{\lambda_{m}}\right)^{v_{m}}\right] \\
= & \frac{\left(-\lambda_{m}\right)^{-v_{m}}}{\Gamma\left(v_{m}\right)} e^{-\frac{y}{\lambda_{m}}} \sum_{r_{m}=0}^{v_{m}-1} C_{v_{m}-1}^{r_{m}} \\
& \times(-y)^{v_{m}-1-r_{m}} \phi^{\left(r_{m}\right)}\left(\lambda_{m}\right)
\end{aligned}
$$

where $\mathrm{C}_{t}^{i}=\frac{t !}{i !(t-i) !}$ denotes the number of ways of selecting $i$ elements from $t$ elements; $C_{t}^{i}=1$ for $i=0$ and $t \geq 0$. Moreover, $\phi^{\left(r_{m}\right)}\left(\lambda_{m}\right)$ is the $r_{m}^{\text {th }}$ order derivative of $\phi\left(\lambda_{m}\right)$, and $\phi\left(\lambda_{m}\right)$ is specified as

$$
\phi\left(\lambda_{m}\right)=\left\{\begin{array}{l}
1, M_{p}=1 \\
\prod_{\substack{n=1 \\
n \neq m}}^{M_{p}}\left(\frac{\lambda_{m}}{\lambda_{m}-\lambda_{n}}\right)^{v_{n}}, M_{p}>1 .
\end{array}\right.
$$

$$
\mathbf{R}=E\left[\alpha \alpha^{H}\right]=\operatorname{diag}\{\underbrace{P_{1} \mu_{1}, \ldots, P_{1} \mu_{1}}_{N^{2-q} \text { same terms }}, \underbrace{P_{2} \mu_{2}, \ldots, P_{2} \mu_{2}}_{N^{2-q} \text { same terms }}, \ldots, \underbrace{P_{K} \mu_{K}, \ldots, P_{K} \mu_{K}}_{N^{2-q} \text { same terms }}\},
$$

$$
\mathbf{Q}=\operatorname{diag}\{\underbrace{\sqrt{P_{1} \mu_{1}}, \ldots, \sqrt{P_{1} \mu_{1}}}_{N^{2-q} \text { same terms }}, \underbrace{\sqrt{P_{2} \mu_{2}}, \ldots, \sqrt{P_{2} \mu_{2}}}_{N^{2-q} \text { same terms }}, \ldots, \underbrace{\sqrt{P_{K} \mu_{K}}, \ldots, \sqrt{P_{K} \mu_{K}}}_{N^{2-q} \text { same terms }}\} .
$$


Using the logarithmic derivative [28] to evaluate the derivatives of $\phi\left(\lambda_{m}\right)$, we have

$$
\phi^{\left(r_{m}\right)}\left(\lambda_{m}\right)=G^{\left(r_{m}\right)}\left(\lambda_{m}\right) \phi\left(\lambda_{m}\right)
$$

where $G^{\left(r_{m}\right)}\left(\lambda_{m}\right)\left(m=1, \ldots, M_{p}\right)$ is equal to 1 when $r_{m}=0$, and is equal to 0 when $r_{m} \geq 1$ and $M_{p}=1$; when $r_{m} \geq 1$ and $M_{p}>1$, it is given as

$\sum_{r_{m}^{\prime}=0}^{r_{m}-1} C_{r_{m}-1}^{r_{m}^{\prime}} g^{\left(r_{m}-1-r_{m}^{\prime}\right)}\left(\lambda_{m}\right) \sum_{r_{m}^{\prime \prime}=0}^{r_{m}^{\prime}-1} C_{r_{m}^{\prime}-1}^{r_{m}^{\prime \prime}} g^{\left(r_{m}^{\prime}-1-r_{m}^{\prime \prime}\right)}\left(\lambda_{m}\right) \times \cdots$.

In (40), $g^{(t)}\left(\lambda_{m}\right)\left(m=1, \ldots, M_{p}\right)$ is further specified as

$$
g^{(t)}\left(\lambda_{m}\right)=t ! \times \sum_{\substack{n=1 \\ n \neq m}}^{M_{p}} \frac{v_{n} \times \lambda_{n}^{t+1} \times \lambda_{m}^{t+1}}{\left(\lambda_{m}-\lambda_{n}\right)^{t+1}} .
$$

By combining (37)-(41), the closed-form expression for the PDF of $Y$ is given as

$$
\begin{aligned}
& f_{Y}(y)=\sum_{m=1}^{M_{p}}\left\{\frac{-e^{\frac{-y}{\lambda_{m}}} \phi\left(\lambda_{m}\right)}{\left(-\lambda_{m}\right)^{v_{m}}} \Gamma\left(v_{m}\right)\right. \sum_{r_{m}=0}^{v_{m}-1} \mathrm{C}_{\boldsymbol{v}_{m}-1}^{r_{m}} \\
&\left.\times(-y)^{v_{m}-1-r_{m}} G^{\left(r_{m}\right)}\left(\lambda_{m}\right)\right\}
\end{aligned}
$$

\section{SIR Distributions}

1) Interfering Links Use Category I MIMO Modes: For the general scenarios where $K>1$ and $P_{i} \mu_{i} \neq P_{j} \mu_{j}$ for $i \neq j$ with $i, j \in\{1, \ldots, K\}$, obviously, $q=1, M_{p}=K$ and $v_{k}=$ $N\left(k=1, \ldots, M_{p}\right)$. Then, the PDF of the postprocessing SIR, $\gamma=\frac{X}{\left\{\frac{1}{N} \cdot Y\right\}}(\gamma>0)$, is given as [30]

$$
\begin{aligned}
f_{\gamma}(\gamma) & =\frac{1}{N} \int_{-\infty}^{+\infty} f_{X}\left(\frac{y \gamma}{N}\right) f_{Y}(y)|y| d y \\
& =\frac{1}{N} \int_{0}^{+\infty} f_{X}\left(\frac{y \gamma}{N}\right) f_{Y}(y) y d y .
\end{aligned}
$$

Due to the complicated mathematical form for $f_{Y}(y)(42)$, a closed-form expression for (43) is not easy to obtain. In practice, numerical computations can be used to evaluate it. But in some specific cases, $f_{Y}(y)$ can be simplified and/or a closed-form expression of (43) can be obtained.

- Special Case I: When the number of transmitting antennas for the interfering BSs, $N$, is equal to 2 , then using (38) and (40)-(42), we get

$$
f_{Y}(y)=\sum_{k=1}^{K}\left\{\frac{-e^{\frac{-y}{P_{k} \mu_{k}}}}{\left(P_{k} \mu_{k}\right)^{2}} \prod_{\substack{j=1 \\ j \neq k}}^{K}\left(\varepsilon_{k}\right)^{2}\left(-y+\sum_{\substack{j=1 \\ j \neq k}}^{K} 2 \varepsilon_{k} P_{j} \mu_{j}\right)\right\}
$$

where $\varepsilon_{k}=\frac{P_{k} \mu_{k}}{P_{k} \mu_{k}-P_{j} \mu_{j}}, K>1$ and $P_{i} \mu_{i} \neq P_{j} \mu_{j}$ for $i \neq j$ with $i, j \in\{1, \ldots, K\}$.
- Special Case II: (i) The number of interfering BSs, $K$, is equal to 1 or (ii) $K>1$ with $P_{1} \mu_{1} \cdots=P_{K} \mu_{K}$; clearly, $M_{p}=1$ and $\lambda_{1}=P_{1} \mu_{1}$ with multiplicity $v_{1}=N K$. Then, using (38) and (40)-(42), we get

$$
f_{Y}(y)=\frac{(y)^{N K-1} \exp \left(\frac{-(y)}{P_{1} \mu_{1}}\right)}{\left(P_{1} \mu_{1}\right)^{N K} \Gamma(N K)},
$$

and, using (29) and (43), $f_{\gamma}(\gamma)$ is given as

$$
\begin{aligned}
& f_{\gamma}(\gamma) \approx \frac{1}{\zeta N \frac{P_{0} \mu_{0}}{P_{1} \mu_{1}}} \\
& \cdot \frac{\Gamma(N M+N K)\left(\frac{\gamma}{\left(\zeta N \frac{P_{0} \mu_{0}}{P_{1} \mu_{1}}\right)}\right)^{N M-1}}{\Gamma(N M) \Gamma(N K)\left(1+\frac{\gamma}{\left(\zeta N \frac{P_{0} \mu_{0}}{P_{1} \mu_{1}}\right)}\right)^{N M+N K}} .
\end{aligned}
$$

2) Interfering Links Use Category II MIMO Modes: For the general scenarios where $K>1$ and $P_{i} \mu_{i} \neq P_{j} \mu_{j}$ for $i \neq j$ with $i, j \in\{1, \ldots, K\}, q=2, M_{p}=K, \lambda_{k}=P_{k} \mu_{k}$ and $v_{k}=1\left(k=1, \ldots, M_{p}\right)$. Then, using (38) and (40)-(42), we get

$$
f_{Y}(y)=\sum_{k=1}^{K}\left\{\frac{e^{\frac{-y}{P_{k} \mu_{k}}}}{P_{k} \mu_{k}} \prod_{\substack{j=1 \\ j \neq k}}^{K} \frac{P_{k} \mu_{k}}{P_{k} \mu_{k}-P_{j} \mu_{j}}\right\} .
$$

Denote the CDF of the postprocessing SIR, $\gamma=\frac{X}{Y}(\gamma \geq 0)$, as $F_{\gamma}(r)=\operatorname{Pr}\{\gamma<r\}$. Then

$$
\begin{aligned}
F_{\gamma}(r) & =\operatorname{Pr}\left\{\frac{X}{Y}<r\right\}=\operatorname{Pr}\left\{Y>\frac{X}{r}\right\} \\
& =\int_{0}^{+\infty} f_{X}(x) \int_{\frac{x}{r}}^{+\infty} f_{Y}(y) d y d x .
\end{aligned}
$$

Because $X \triangleq\left|d_{0,1}\right|^{2}$ is also a central quadratic form in i.i.d. complex Gaussian RVs with zero mean and variance $P_{0} \mu_{0}$, for $X, M_{p}=1$ and $\lambda_{1}=\zeta P_{0} \mu_{0}$ with $v_{m}=N M$. From (35), we get the MGF of $X$ as

$$
\Phi_{X}(s)=E\left[e^{s x}\right]=\frac{1}{\left(1-s \times \zeta P_{0} \mu_{0}\right)^{N M}} .
$$

Furthermore, we denote $\prod_{j=1, j \neq k}^{K} \frac{P_{k} \mu_{k}}{P_{k} \mu_{k}-P_{j} \mu_{j}}$ as $\xi_{k}$; by utilizing the fact that $\int_{0}^{\infty} f_{Y}(y) d y=1$, we can easily obtain $\sum_{k=1}^{K} \xi_{k}=$ 1 . Then, using (47), (48) becomes

$$
\begin{aligned}
F_{\gamma}(r) & =\int_{0}^{+\infty} f_{X}(x) \int_{\frac{x}{r}}^{+\infty} \sum_{k=1}^{K} \frac{\xi_{k}}{P_{k} \mu_{k}} \exp \left(-\frac{y}{P_{k} \mu_{k}}\right) d y d x \\
& =\int_{0}^{+\infty} f_{X}(x) \sum_{k=1}^{K} \xi_{k} \exp \left(-\frac{x}{r P_{k} \mu_{k}}\right) d x \\
& =\sum_{k=1}^{K} \xi_{k} \int_{0}^{+\infty} \exp \left(-\frac{1}{r P_{k} \mu_{k}} x\right) f_{X}(x) d x \\
& =\sum_{k=1}^{K} \xi_{k} \cdot E\left[\exp \left(-\frac{1}{r P_{k} \mu_{k}} x\right)\right]
\end{aligned}
$$


By replacing (49) with $s=\frac{-1}{\left(r P_{k} \mu_{k}\right)}$, the CDF of the postprocessing SIR is

$$
F_{\gamma}(r)=\sum_{k=1}^{K} \frac{\xi_{k}}{\left(1+\frac{\zeta}{r} \frac{P_{0} \mu_{0}}{P_{k} \mu_{k}}\right)^{N M}} .
$$

By differentiating (51), we get the PDF of the SIR as

$$
f_{\gamma}(\gamma)=\sum_{k=1}^{K} \xi_{k} \cdot\left(\frac{P_{k} \mu_{k}}{\zeta P_{0} \mu_{0}}\right) \cdot \frac{(N M)\left(\frac{P_{k} \mu_{k}}{\zeta P_{0} \mu_{0}} \gamma\right)^{N M-1}}{\left(1+\frac{P_{k} \mu_{k}}{\zeta P_{0} \mu_{0}} \gamma\right)^{N M+1}}, \gamma>0
$$

- Special Case: i) the number of interfering BSs, $K$, is equal to 1 or ii) $K>1$ with $P_{1} \mu_{1} \cdots=P_{K} \mu_{K}$, clearly, $M_{p}=1$ and $\lambda_{1}=P_{1} \mu_{1}$ with the multiplicity of $v_{1}=K$. Then, using (38) and (40)-(42), we get

$$
f_{Y}(y)=\frac{y^{K-1} \exp \left(\frac{-y}{P_{1} \mu_{1}}\right)}{\left(P_{1} \mu_{1}\right)^{K} \Gamma(K)}
$$

and, using (29) and (43), $f_{\gamma}(\gamma)$ is given as

$$
f_{\gamma}(\gamma) \approx \frac{1}{\zeta \frac{P_{0} \mu_{0}}{P_{1} \mu_{1}}} \cdot \frac{\Gamma(N M+K)\left(\frac{\gamma}{\left(\zeta \frac{P_{0} \mu_{0}}{P_{1} \mu_{1}}\right)}\right)^{N M-1}}{\Gamma(N M) \Gamma(K)\left(\frac{1+\gamma}{\left(\zeta \frac{P_{0} \mu_{0}}{P_{1} \mu_{1}}\right)}\right)^{N M+K}} .
$$

\section{Simulation Results}

In this section, the validity of the theoretical analyses is verified by comparing the analytically obtained PDFs/CDFs with Monte Carlo simulations. All the BSs (including the desired BS) are assumed to be equipped with $N$ antennas and the desired receiver has $M$ antennas. In the Monte Carlo simulations, STBC and MBF/MRT are used to represent Category I and Category II interferers, respectively. For the desired link, $P_{0} \mu_{0}$ is set to 1. The notation $K=6\{1,0.9,0.8,0.7,0.6,0.5\}$ means that there are $K=6$ interfering BSs; the $k$ th number within the braces is the value of $P_{k} \mu_{k}$ for the $k$ th interfering BS. Moreover, in the legends, capitalized "I" and "II" are used to denote "Category I" and "Category II" interfering MIMO modes, respectively; "(A)" and "(S)" represents the "Analytical" and "Monte-Carlo simulation" results, respectively.

First, we verify the validity of the PDF expressions, (42) and (29), in Figs. 1-3. Note that, when an interfering BS has one antenna (i.e., $N=1$ ), only SIMO mode can be used. Hence, for this case, only one-pair of PDF curves appear in Fig. 2. From Figs. 1 and 2, we see that the analytical results of (42) match the corresponding Monte Carlo simulation results perfectly. Although there is some mismatch between (29) and the Monte Carlo results within a small SIR range, the deviation is very small. Another important observation from Figs. 1 and 2 is the remarkable distinction between the PDFs of Category I and II interfering MIMO modes. This distinction results in different impact of the two categories on the desired user.

From Figs. 4-6, we find that the analytical CDFs match the corresponding simulation CDFs very well. Besides the cases of $P_{i} \mu_{i} \neq P_{j} \mu_{j}(i \neq j)$, the case of $P_{i} \mu_{i}=P_{j} \mu_{j}(i \neq j)$ is

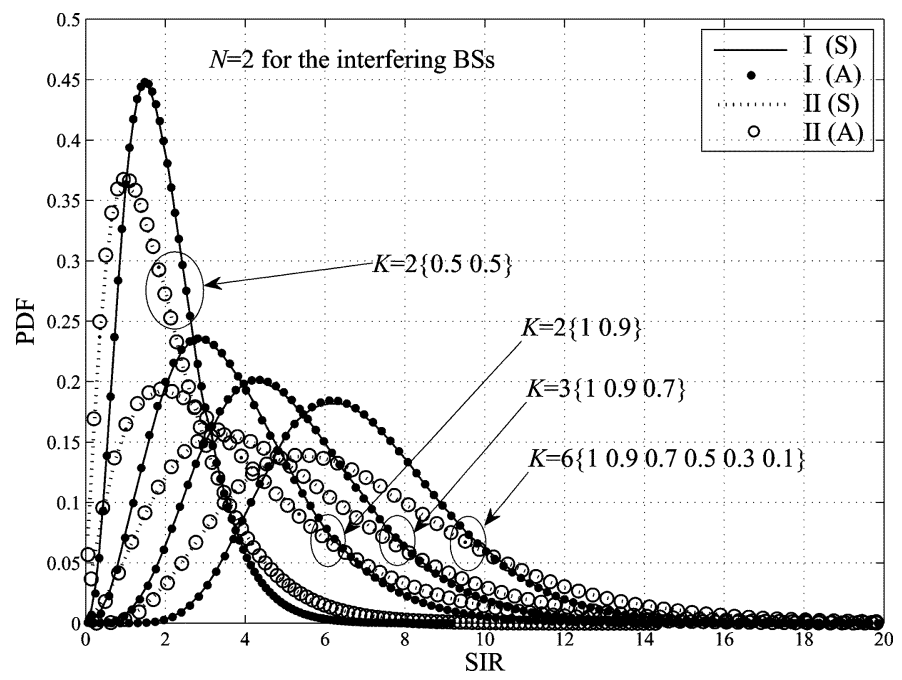

Fig. 1. Validity of the PDF of the interference term in the SIR, (42), with $N=2$.

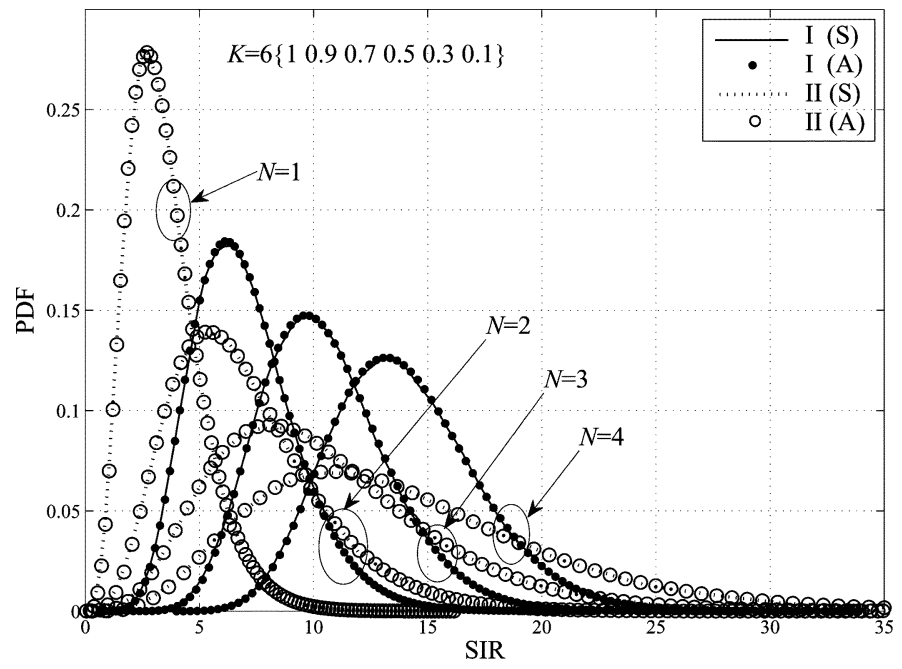

Fig. 2. Validity of the PDF of the interference term in the SIR, (42), with $K=6$.

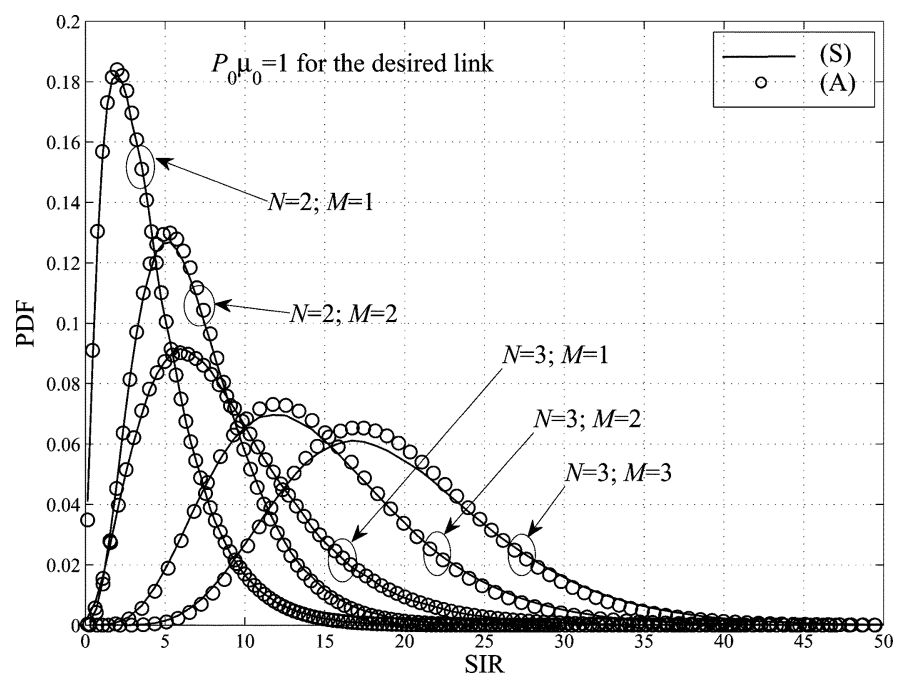

Fig. 3. Validity of the PDF of the desired signal term in the SIR, (29).

verified in Fig. 4. A major observation from Figs. 4-6 is that MIMO modes from different categories have different impact 


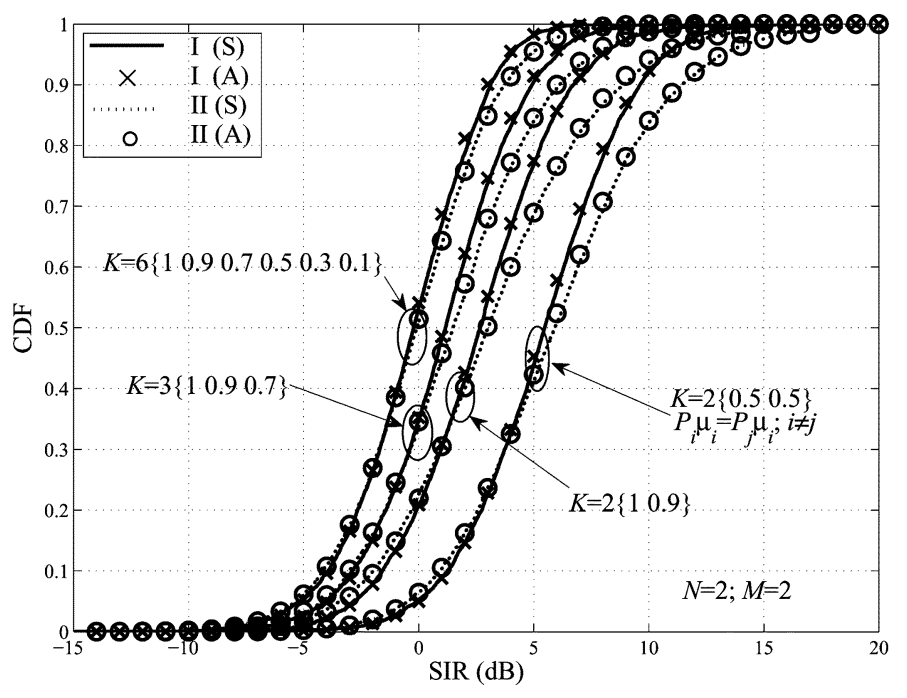

Fig. 4. Impact of total equivalent interfering signal power.

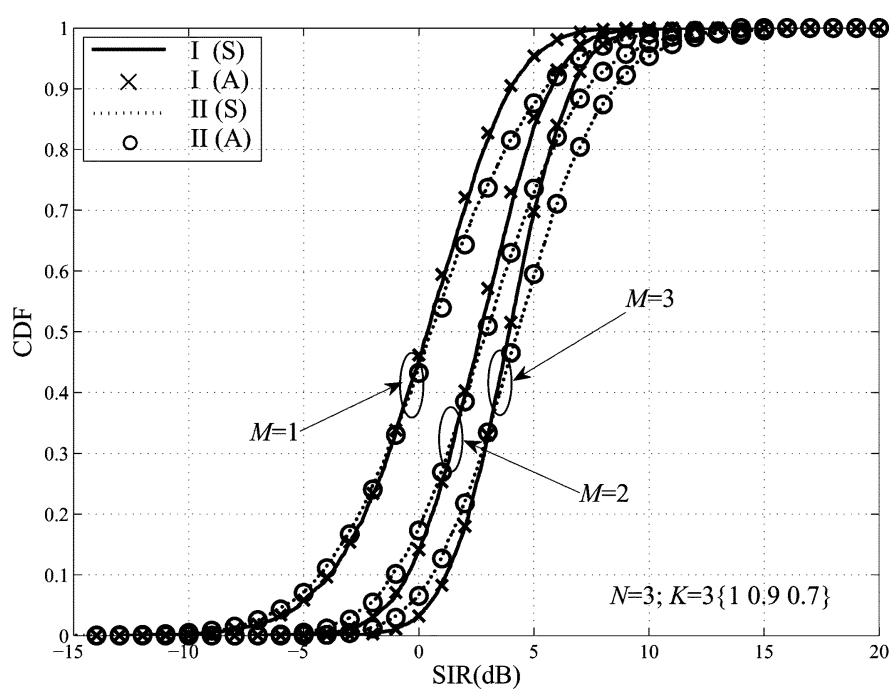

Fig. 5. Impact of the number of receive antennas, $M$, at the desired receiver.

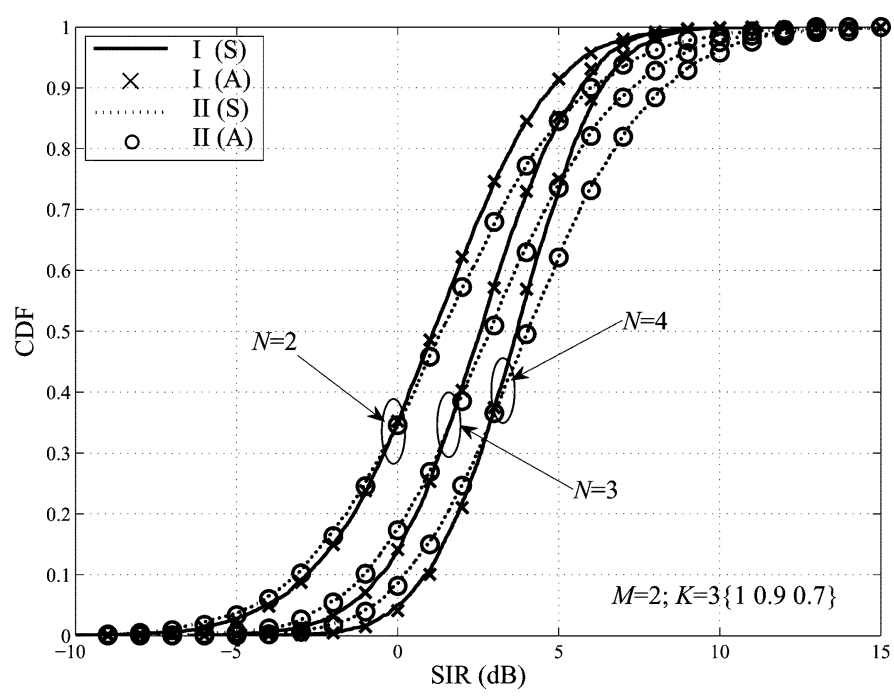

Fig. 6. Impact of the number of transmit antennas, $N$, at the BSs.

on the desired user. Moreover, the CDF curves for the two categories under the same condition have a crossover at some SIR value. Beyond the crossover, the Category II MIMO schemes have less impact on the desired link; below this crossover, Category II has more impact on the desired link.

In Fig. 4, the antenna configuration is fixed and the impact of the total equivalent interfering signal power at the desired receiver is investigated. From the SIR expressions, it is easy to see that the total equivalent interfering signal power is proportional to $\sum_{k=1}^{K} P_{k} \mu_{k}$ for both Category I and II interferers. As expected, when $\sum_{k=1}^{K} P_{k} \mu_{k}$ increases, the performance of the desired link degrades. In Fig. 5, the parameters of the interfering signals ( $K$ and $P_{k} \mu_{k}$ ) and $N$ are fixed. The impact of the number of receiving antennas, $M$, is evaluated. From (22), we see that $M$ only affects the desired received signal. Hence, when $M$ increases (i.e., the diversity gain $N M$ increases), the performance of the desired link improves. The impact of the number of transmit antennas at the BSs, $N$, is shown in Fig. 6. Although $N$ affects both the desired received signal and interfering received signal, the total equivalent interfering power does not change with $N$. Therefore, an increase in $N$ improves the performance of the desired MBF receiver.

Another focus in this section is the performance gap between the two categories. In (22), the mean and variance of the co-channel interference term for Category I are $\sum_{k=1}^{K} P_{k} \mu_{k}$ and $\frac{2}{N} \sum_{k=1}^{K} P_{k} \mu_{k}$, respectively. For Category II interferers, the parameters are $\sum_{k=1}^{K} P_{k} \mu_{k}$ and $2 \sum_{k=1}^{K} P_{k} \mu_{k}$. The performance gap between the two categories arises from the difference in the variances. Hence, when $N$ and $\sum_{k=1}^{K} P_{k} \mu_{k}$ are fixed (see Fig. 5), a change in the number of receive antennas does not have a significant effect on the gap between the two categories. In Fig. 4 , an increase in $\sum_{k=1}^{K} P_{k} \mu_{k}$ when $N$ is fixed will narrow the gap between the variances, hence, the gap between the performance of the two categories decreases. For the case in Fig. 6, the distinction between the variances becomes larger with an increase in $N$; therefore, the performance gap between the two categories increases.

\section{CONCLUSION}

With a realistic channel model, including the effects of pathloss and Rayleigh fading, the statistical distributions of the postprocessing SIRs for MBF with two categories of unequal-power MIMO interferers are derived. The validity of the PDF expressions is verified by Monte Carlo simulation results. Both theoretical analysis and simulation results show that the two categories have different impact on the desired MBF receiver. Moreover, the performance gap between the two categories is mainly determined by the number of transmit antennas.

\section{REFERENCES}

[1] S. Catreux, P. F. Driessen, and L. J. Greenstein, "Simulation results for an interference-limited multiple-input multiple-output cellular system," IEEE Commun. Lett., vol. 4, no. 11, pp. 334-336, Nov. 2000.

[2] A. Goldsmith, Wireless Communications. Cambridge, U.K.: Cambridge Univ. Press, 2005.

[3] S. M. Alamouti, "A simple transmit diversity technique for wireless communications," IEEE J. Sel. Areas Commun., vol. 16, no. 8, pp. 1451-1458, Oct. 1998.

[4] V. Tarokh, N. Seshadri, and A. R. Calderbank, "Space-time codes for high data rate wireless communication: Performance criterion and code construction," IEEE Trans. Inf. Theory, vol. 44, no. 2, pp. 744-765, Mar. 1998. 
[5] T. Lo, "Maximal ratio transmission," IEEE Trans. Commun., vol. 47, no. 10 , pp. $1458-1461$, Oct. 1999.

[6] J. Cui and A. U. H. Sheikh, "Outage probability of cellular radio systems using maximal ratio combining in the presence of multiple interferers," IEEE Trans. Commun., vol. 47, no. 8, pp. 1121-1124, Aug. 1999.

[7] V. A. Aalo and C. Chayawan, "Outage probability of cellular radio systems using maximal ratio combining in Rayleigh fading channel with multiple interferers," Electron. Lett., vol. 36, no. 15, pp. 1314-1315, Jul. 2000.

[8] J. M. Romero-Jerez, J. P. P. Martin, and A. J. Goldsmith, "Outage probability of MRC with arbitrary power cochannel interferers in Nakagami fading," IEEE Trans. Commun., vol. 55, no. 7, pp. 1283-1286, Jul. 2007.

[9] C. Chayawan and V. A. Aalo, "Average error probability of digital cellular radio systems using MRC diversity in the presence of multiple interferers," IEEE Trans. Wireless Commun., vol. 2, no. 5, pp. 860-864, Sep. 2003.

[10] Y. Akyildiz and B. D. Rao, "Statistical performance analysis of optimum combining with co-channel interferers and flat Rayleigh fading," in Proc. IEEE GLOBECOM, Nov. 25-29, 2001, vol. 6, pp. 3663-3667.

[11] A. Shah and A. Haimovich, "Performance analysis of maximal ratio combining and comparison with optimum combining for mobile radio communications with cochannel interference," IEEE Trans. Veh. Technol., vol. 49, no. 4, pp. 1454-1463, Jul. 2000.

[12] W. Choi, N. Himayat, S. Talwar, and M. Ho, "The effects of co-channel interference on spatial diversity techniques," in Proc. IEEE Wireless Commun. Netw. Conf. (WCNC), Mar. 11-15, 2007, pp. 1936-1941.

[13] M. Rahman, E. de Carvalho, and R. Prasad, "Impact of MIMO co-channel interference," in Proc. IEEE Int. Symp. IEEE Personal, Indoor, Mobile Radio (PIMRC), Sep. 3-7, 2007, pp. 1-5.

[14] Y. Li, L. Cimini, and N. Himayat, "Performance analysis of space time block coding with co-channel MIMO interferers," in Proc. IEEE GLOBECOM, Dec. 4, 2008, pp. 1-5.

[15] M. Kang and M.-S. Alouini, "A comparative study on the performance of MIMO MRC systems with and without cochannel interference," IEEE Trans. Commun., vol. 52, no. 8, pp. 1417-1425, Aug. 2004.

[16] L. Yang and J. Qin, "Outage performance of MIMO MRC systems with unequal-power co-channel interference," IEEE Commun. Lett., vol. 10, no. 4, pp. 245-247, Apr. 2006.

[17] J. M. Romero-Jerez, J. P. Pena-Martin, G. Aguilera, and A. J. Goldsmith, "Performance of MIMO MRC systems with co-channel interference," in Proc. IEEE Int. Commun. Conf. (ICC), Jun. 2006, vol. 3, pp. 1343-1349.

[18] J. P. Pena-Martin, J. M. Romero-Jerez, G. Aguilera, and A. Goldsmith, "Performance comparison of MRC and IC under transmit diversity," IEEE Trans. Wireless Commun., vol. 8, no. 5, pp. 2484-2493, May 2009.

[19] X. Rui, R. Jin, and J. Geng, "Performance analysis of MIMO MRC systems in the presence of self-interference and co-channel interferences," IEEE Signal Process. Lett., vol. 14, no. 11, pp. 801-803, Nov. 2007.

[20] K. S. Ahn, "Performance analysis of MIMO-MRC systems with channel estimation error in the presence of cochannel interferences," IEEE Signal Process. Lett., vol. 15, pp. 445-448, 2008.

[21] W. Zhang, Z. Guan, and X. Zhou, "Analysis of SER of MIMO-MRC systems with imperfect channel estimation in the presence of non-Rayleigh CCIs," Int. J. Commun. Syst., pp. 1-5, Nov. 2010.

[22] K. T. Fang and Y. T. Zhang, Generalized Multivariate Analysis. New York: Springer, 1990.

[23] P. A. Dighe, R. K. Mallik, and S. S. Jamuar, "Analysis of transmitreceive diversity in Rayleigh fading," IEEE Trans. Commun., vol. 51, no. 4, pp. 694-703, Apr. 2003.

[24] E. Sengul, E. Akay, and E. Ayanoglu, "Diversity analysis of single and multiple beamforming," IEEE Trans. Commun., vol. 54, no. 6, pp. 990-993, Jun. 2006.

[25] J. Choi, "Performance analysis of the closed-loop transmit antenna diversity system over Rayleigh fading channels," IEEE Trans. Veh. Tech., vol. 51, no. 4, pp. 767-771, Jul. 2002.

[26] M. Wennstrom, M. Helin, A. Rydberg, and T. Oberg, "On the optimality and performance of transmit and receive space diversity in MIMO channels," in IEE Seminar on MIMO: Communications Systems from Concept to Implementations (Ref./175), Dec. 2001, pp. 4/1-4/6.

[27] J. B. Andersen, "Array gain and capacity for known random channels with multiple element arrays at both ends," IEEE J. Sel. Areas Commun., vol. 18, no. 11, pp. 2172-2178, Nov. 2000.

[28] W. Conradie and A. Gupta, "Quadratic forms in complex normal variates: Basic results," Statistica, no. 47, pp. 73-84, 1987.

[29] J. Brown and R. Churchill, Complex Variables and Applications. New York: McGraw-Hill, 2008.

[30] M. DeGroot and M. Schervish, Probability and Statistics, 3rd ed. Reading, MA: Addison-Wesley, 2001.

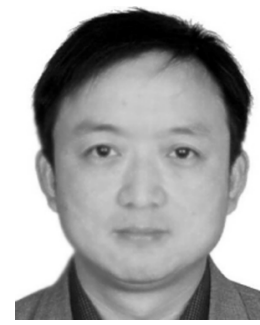

communications, etc.

In 2008, he received the Best Paper Award of the IEEE ChinaCOM international conference. He has been funded by more than ten projects, including the National Natural Science Foundation of China, RCUK for the UK-China Science Bridges Project, the Special Grade of China Postdoctoral Science Foundation funded project, and the Important National Science \& Technology Specific Projects.

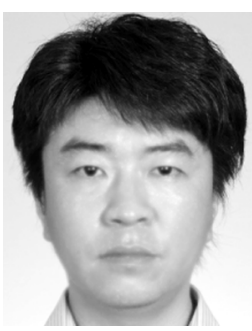

Lu Zhang (S'06-M'09) was born in China. He received the M.E. and B.E. degrees from the Department of Electronic Engineering, Beijing Institute of Technology, China, in 2002 and 1999, respectively, and the Ph.D. degree from the Department of Electrical and Computer Engineering, University of Delaware, Newark, in 2009.

From April 2002 to January 2004, he was an R\&D Engineer of the Wireless Communications Department in the Beijing Research Center of HuaWei Technologies Limited Company. From January 2004 to August 2004, he was a System Engineer of the Information and Communication Mobile Networks Department in Siemens Ltd., China. From November 2008 to March 2009, he was a full-time Research Intern of Mitsubishi Electric Research Laboratories (USA). He has been a Research Scientist at Alcatel-Lucent Bell Labs, China, since 2009. His research interests include interference management for small cells and/or heterogeneous networks in LTE-Advanced systems, theoretical analyses for multi-cell co-channel interference in MIMO-based cellular networks, decentralized cooperative relaying techniques in ad hoc networks (including decentralized distributed STBC technique, decentralized relay management strategy, efficient power allocation, multihop routing with minimal control overhead, multi-source communication with minimal control overhead), and space-time coding in MIMO systems.

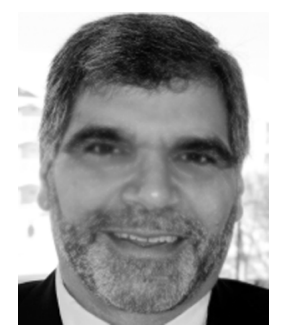

Leonard J. Cimini, Jr. (S'77-M'82-SM'89-F'00) received the $\mathrm{Ph} . \mathrm{D}$. degree from the University of Pennsylvania, in 1982.

He worked at AT\&T, first in Bell Labs and then AT\&T Labs, for 20 years. He has been a Professor at the University of Delaware, Newark, since 2002.

Dr. Cimini began his ComSoc activities 25 years ago in the Communication Theory Technical Committee. He is currently VP-Publications, and, among other publications-related positions, is the founding Editor-in-Chief of the IEEE JOURNAL ON SElECTED AREAS In COMMUNICATIONS: Wireless Communications Series. He was elected an IEEE Fellow in 2000 for contributions to the theory and practice of high-speed wireless communications. For this pioneering work, he was given the 2007 James R. Evans Avant Garde Award from the IEEE Vehicular Technology Society and the 2010 Innovators Award from the NJ Inventors Hall of Fame. In 2010, he received several ComSoc awards, including the Stephen O. Rice Prize, the Donald W. McLellan Meritorious Service Award, and the Recognition Award from the Wireless Communications Technical Committee. 


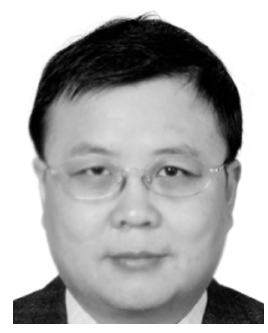

Hailin Zhang (M'98) received the B.S. and M.S. degrees from Northwestern Polytechnic University, Xi'an, China, in 1985 and 1988, respectively, and the Ph.D. form Xidian University, Xi'an, China, in 1991, all in electronic information engineering.

Since then, he has been with Xidian University as a member of teaching and researching and is currently a senior Professor and a Ph.D. adviser with School of Telecommunications Engineering at Xidian University. He is currently the Dean of this School, the Director of Key Laboratory in Wireless Communica- tions Sponsored by China Ministry of Information Technology, a key member of State Key Laboratory of Integrated Services Networks, one of the state government specially compensated scientists and engineers, a field leader in Telecommunications and Information Systems in Xidian University, and an Associate Director for National 111 Project. His current research interests include key transmission technologies and standards on broadband wireless communications for B3G, 4G, and next-generation broadband wireless access systems. He has recently published 78 papers in core journals and conferences. 